Journal of Applied Fluid Mechanics, Vol. 14, No. 6, pp. 1755-1765, 2021. Available online at www.jafmonline.net, ISSN 1735-3572, EISSN 1735-3645. https://doi.org/10.47176/jafm.14.06.32493

\title{
Boundary Condition Effect on Two-Phase Fluid Flow and Heat Transfer inside 3-D Microchannels
}

\author{
S. Chandrasekhar and V. R. K. Raju ${ }^{\dagger}$ \\ Department of Mechanical Engineering, National Institute of Technology Warangal, \\ Telangana State, India - 506004 \\ $\dagger$ Corresponding Author Email: vrkraju@nitw.ac.in
}

(Received December 20, 2020; accepted June 5, 2021)

\begin{abstract}
Two-phase Taylor flows play a vital role in dissipating heat effectively for the proper functioning of electronic systems. In the present study, the thermal performance of liquid-liquid Taylor flow was carried out in a 3D microchannel with uniform wall heat flux boundary for five different cases: uniform heat flux on the four walls, three walls, two opposite walls, single wall, and two adjacent walls, and the aspect ratio of microchannel was varied in the range of 0.2-5. The length of the microchannel was $4 \mathrm{~mm}$, height and width were $0.1 \mathrm{~mm}$ each for a square microchannel. For varying aspect ratios of the microchannel, the height and width of the microchannel were taken in the range of 0.06-0.3 mm to keep the hydraulic diameter constant. Dodecane and water were the working fluids in the study and assumed to be Newtonian, incompressible, immiscible, and the properties of fluids were assumed to be independent of temperature. The pressure distribution in the microchannel was investigated under five thermal boundary cases and the aspect ratio effect on pressure drop was also discussed. Results showed Nusselt number of two-phase flow with four-wall heat flux increases up to $280 \%$ compared to liquid-only flow and it has been validated with standard heat transfer correlation available in the literature. A higher heat transfer rate $(N u=10.41)$ was recorded in the opposite walls boundary condition and the heat transfer rate $(N u=7.81)$ was minimum when the adjacent walls were subjected to uniform heat flux. The effect of microchannel aspect ratio on Taylor flow heat transfer under thermal boundary conditions was also analyzed.
\end{abstract}

Keywords: Taylor flow; Aspect ratio; Heat transfer; CFD.

\section{NOMENCLATURE}

$\begin{array}{ll}\mathrm{D}_{\mathrm{h}} & \text { hydraulic diameter }(\mathrm{m}) \\ \mathrm{e} & \text { energy }(\mathrm{J} / \mathrm{Kg}) \\ \mathrm{h} & \text { enthalpy }(\mathrm{J} / \mathrm{Kg}) \\ \mathrm{h}_{\mathrm{c}} & \text { heat transfer coefficient }\left(\mathrm{W} / \mathrm{m}^{2}-\mathrm{K}\right) \\ \mathrm{k} & \text { thermal conductivity }(\mathrm{W} / \mathrm{m}-\mathrm{K}) \\ \mathrm{L}_{\mathrm{s}} & \text { slug length }(\mathrm{m}) \\ \mathrm{P} & \text { pressure }(\mathrm{Pa}) \\ q_{\mathrm{w}} & \text { wall heat flux }\left(\mathrm{W} / \mathrm{m}^{2}\right) \\ \mathrm{t} & \text { time }(\mathrm{s}) \\ \mathrm{T}_{\mathrm{b}} & \text { bulk temperature }(\mathrm{K}) \\ \mathrm{T}_{\mathrm{w}} & \text { wall temperature }(\mathrm{K})\end{array}$

\section{INTRODUCTION}

Nowadays microchannels are extensively used in various applications like micro-reactors, lab on chip, microelectronics, and heat exchangers. Earlier, single-phase flows were used to remove heat from electronic systems, but such single-phase flows were limited to low heat fluxes. Recently, two-phase flows were introduced to remove higher heat fluxes. The

$\begin{array}{ll}\mathrm{Ca} & \text { Capillary number } \\ \mathrm{Nu} & \text { Nusselt number } \\ \mathrm{Pr} & \text { Prandtl number } \\ \mathrm{Re} & \text { Reynolds number } \\ v & \text { velocity vector }(\mathrm{m} / \mathrm{s}) \\ \alpha_{\mathrm{d}} & \text { volume fraction } \\ \rho & \text { density }\left(\mathrm{kg} / \mathrm{m}^{3}\right) \\ \mathrm{K} & \text { interface curvature } \\ \sigma & \text { surface tension }(\mathrm{N} / \mathrm{m}) \\ \mu & \text { viscosity }(\mathrm{kg} / \mathrm{m}-\mathrm{s})\end{array}$

potentiality of these flows over single-phase flows has been studied and reported by several researchers (Asadolahi et al. 2011; Bandara et al. 2015; Dai et al. 2015; Muzychka et al. 2010; Triplett et al. 1999; Leung et al. 2010). Two-phase flows exhibit different flow patterns when two immiscible fluids are pushed into a microchannel at different flow rates. The flow patterns generated include bubbly flow, churn flow, slug flow, and dispersed bubbly 
flow. Among them, Taylor/slug flows have attracted several researchers because these flows are easy to generate when the surface tension forces overcome gravitational force. In Taylor flows, a series of dispersed slugs are separated by continuous carrier fluid at regular intervals, and these dispersed phases are separated by a thin film from the channel wall.

Numerous researches have been done on two-phase Taylor flows on the hydrodynamic and thermal characteristics in terms of bubble velocity (Liu et al. 2005), bubble shape (Triplett et al. 1999; Leung et al. 2012), slug length (Leung et al. 2012), film thickness (Bretherton 1961; Irandoust and Andersson 1989; Aussillous and Quere 2000), pressure drop (Warnier et al. 2010; Kreutzer et al. 2005), and thermal characteristics (Che et al. 2012; Dai et al. 2015; Leung et al. 2010; Walsh et al. 2010). The bubble/droplet shape, pressure drop, and heat transfer rates depend on the thin film between the dispersed phase and channel wall. Several theoretical correlations have been developed to measure the thin film as a function of capillary number (Bretherton 1961; Irandoust and Andersson 1989; Aussillous and Quere 2000). Eain et al. (2013) and Han and Shikazono (2009) developed film thickness correlations based on the capillary and weber numbers. Pressure drop is a vital role in microchannel design and flow stability, and the pressure drop correlations were developed using experimental and analytical methods in the twophase flow (Kreutzer et al. 2005; Jovanović et al. 2011; Gupta et al. 2013). In most of the two-phase flows studies, gas bubbles are considered to be dispersed phase, but the role of gas-phase in heat transfer augmentation is negligible in gas-liquid Taylor flows. However, the liquid droplets have higher thermophysical properties vis-a-vis gaseous bubbles, therefore the liquid-liquid Taylor flow was introduced by replacing gaseous bubbles with immiscible liquid droplets.

Urbant et al. (2008) examined the thermal performance of liquid-liquid flow in micro-tubes and the study revealed that internal circulation within the dispersed phase liquid and the distortion in the carrier fluid phase improved the rate of heat transfer. Asthana et al. (2011) experimentally investigated the thermal behavior of both single-phase and twophase flows in a serpentine microchannel with isothermal boundary condition. Results showed that the Nusselt number of two-phase flow increases by four times over liquid-only flow. Eain et al. (2015) examined the local Nusselt number augmentation with uniform heat flux boundary conditions. They observed that there was an enhancement of up to $600 \%$ in the liquid-liquid Taylor flow and they also found that the heat transfer increases with an increase in droplet length and decrease in slug length. Dai et al. (2015) used both experimental and numerical techniques to explore the hydrodynamics and thermal performance of two-phase Taylor flow. They also developed a correlation to predict the thermal performance of two-phase flows. Che et al. (2015) numerically examined the impact of Peclet number, droplet length, and aspect ratio of the rectangular microchannel on thermal behavior of Taylor flow with isothermal wall boundaries. Results indicate that the heat transfer improves with an increase in Peclet number and decreases with increasing AR of the rectangular microchannel. Bandara et al. (2015) explored the thermal characteristics for varying Reynolds numbers (4.9-21.9) for liquid-liquid slug flow with isothermal boundary conditions. They also examined the effect of capillary number, film thickness, slug length, and the contact angle on thermal behavior of slug flow in a circular microchannel.

The nature of the wall boundary condition impacts the thermal performance of the two-phase flows. Vivekanand and Raju (2019) explored the thermal behavior of liquid-liquid Taylor flow, where the modulated thermal boundary condition was applied to channel walls. Results revealed that the heat transfer significantly increases with modulated wall temperature over unmodulated wall temperature boundary condition. Another study by Vivekanand and Raju (2020) examined the thermal performance of Taylor flow under modulated wall motion. They observed 23\% improvement in heat transfer with modulated wall motion compared to unmodulated wall motion. Applying the right wall boundary condition was also very important to dissipate a lot of heat from the channel. Dharaiya and Kandlikar (2012) numerically studied the thermal characteristics of single-phase flow inside the microchannel with varying aspect ratios (AR) in the range of 0.1 to 10 . They performed numerical simulations under uniform heat flux boundary for five different cases: uniform heat flux on four walls, three walls, two opposite walls, a single wall, and two adjacent walls. Results indicate that with an increase in AR the rate of heat transfer increases in the case of three heating walls, and decreases for two opposite and one side wall heating cases. For a square $(\mathrm{AR}=1)$ microchannel, maximum and minimum heat transfer rates were found for two opposite and adjacent heating wall cases respectively.

From the literature, it is observed that thermal characteristics of two-phase flows focused either on uniform heat flux or uniform temperature boundary conditions. Few studies have applied different boundary conditions (modulated wall temperature and motion) to improve heat transfer performance. And a study of single-phase flow was reported the thermal characteristics under five different uniform wall heat flux boundary conditions, but such studies have not been reported in two-phase flows. In order to fill this gap, the present work was carried out to examine the effect of different heating wall boundary conditions on the thermal characteristics of twophase Taylor flow. Further, the present work also analyzed the effect of the aspect ratio on liquid-liquid Taylor flow heat transfer under five different uniform wall boundary cases.

\section{CFD METHODOLOGY}

In the present numerical work, transient simulations were performed on 3D microchannels to explore the liquid-liquid Taylor flow thermal characteristics 
under uniform wall heat flux. The volume of fluid (VOF), phase field, and level set methods mostly used interface tracking methods to capture the interface of working fluids. In this work, VOF method was employed in ANSYS fluent to track the interface of two working fluids. The working fluids were considered to be Newtonian, incompressible, immiscible with the properties of fluids are independent of temperature, while interfacial tension was assumed to be constant.

\subsection{Governing Equations}

The following governing equations (Eq's (1) to (3)) along with volume fraction equation (Eq. (4)) are solved during the simulation by the numerical model (Gupta et al. 2010; Abdollahi et al. 2020).

Continuity:

$\frac{\partial \rho}{\partial t}+\nabla \cdot(\rho v)=0$

Momentum:

$\frac{\partial(\rho v)}{\partial t}+\nabla \cdot(\rho v v)=-\nabla P+\nabla \cdot\left[\mu\left(\nabla v+\nabla v^{T}\right)\right]+F$

Energy:

$\frac{\partial(\rho e)}{\partial t}+\nabla \cdot(\rho v h)=\nabla \cdot(k \nabla T)$

VOF equation:

$$
\frac{\partial \alpha}{\partial t}+v \cdot \nabla \alpha=0
$$

The volume fraction weighted-average method was used to determine the bulk properties, such as dynamic viscosity $(\mu)$, density ( $\rho$ ), and thermal conductivity $(k)$ of the two-phase flow. A model was suggested by Brackbill and Kothe (1992) to evaluate the continuum surface force (CSF). The CSF in the present model is calculated by incorporating the following equation (Eq. (5)) in ANSYS fluent as follows;

$F=\sigma \kappa \frac{\rho \nabla \alpha_{d}}{0.5\left(\rho_{c}+\rho_{d}\right)}$

$\mathrm{K}$ is the interface curvature and is defined in terms of the divergence of the interface normal $(\hat{n})$,

$\kappa=\nabla \hat{n}$

Where $\hat{n}=\frac{n}{|n|}$ and $\mathrm{n}$ is the normal vector

\subsection{Computational Domain and Boundary Conditions}

Figure 1 shows the schematic representation of 3D microchannel (aspect ratio $=1$ ). The length of the microchannel was $4 \mathrm{~mm}$, while the height and width were $0.1 \mathrm{~mm}$ each. For varying aspect ratios of the microchannel, the height and width were taken in the range of 0.06-0.3 mm to keep the hydraulic diameter constant. At the inlet boundary, two-phase velocity $0.2 \mathrm{~m} / \mathrm{s}$ and at the outlet boundary gauge pressure of $0 \mathrm{~Pa}$ were considered. Dodecane and water were taken as primary and secondary fluids, respectively and the properties of working fluids are listed in Table 1. The length of the droplet and liquid slug were 200 and 250 microns respectively. A uniform wall heat flux of $30 \mathrm{~kW} / \mathrm{m}^{2}$ was applied to microchannel walls.

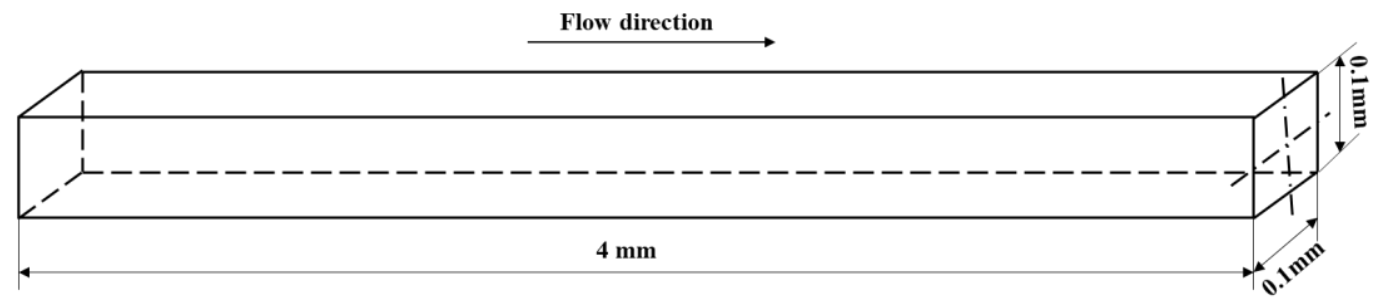

Fig. 1. Schematic of the 3D microchannel $(A R=1)$.

Table 1 Properties of working fluids

\begin{tabular}{|c|c|c|c|c|c|}
\hline Properties & $\begin{array}{c}\text { Viscosity } \\
(\mathrm{kg} / \mathrm{m}-\mathrm{s})\end{array}$ & $\begin{array}{c}\text { Density } \\
\left(\mathrm{kg} / \mathrm{m}^{3}\right)\end{array}$ & $\begin{array}{c}\text { Specific heat } \\
(\mathrm{J} / \mathrm{kg}-\mathrm{K})\end{array}$ & $\begin{array}{c}\text { Thermal conductivity } \\
(\mathrm{W} / \mathrm{m}-\mathrm{K})\end{array}$ & $\begin{array}{c}\text { Interfacial Tension } \\
(\mathrm{N} / \mathrm{m})\end{array}$ \\
\hline Water & 0.00091 & 996.1 & 4070 & 0.61 & ---- \\
\hline Dodecane & 0.00139 & 754.3 & 2210 & 0.13 & 0.0520 \\
\hline
\end{tabular}

\subsection{Numerical Schemes}

The governing equations were solved during CFD simulations by employing VOF model. An explicit geometric reconstruction approach was adopted to solve VOF equation with a maximum courant number of 0.25 . The first-order non-iterative fractional step scheme was employed to solve the unsteady terms of the flow equations. To solve the Pressure, Poisson equation body force weighted technique was used, and to discretize the momentum and energy equations, QUICK scheme was employed. The scalar gradients were determined using Green-Gauss node-based technique to reduce 
spurious currents induced by the discretization of the surface tension term (Gupta et al. 2009). A variable time-stepping method was used with time step size in the range of $10^{-9}-10^{-6}$.

\section{RESULTS AND DISCUSSIONS}

\subsection{Mesh Independence and Validation of the Work}

The techniques recommended by Gupta et al. (2009) and Asadolahi et al. (2011) were implemented to discretize the computational domain in this numerical study. The mesh for the domain was generated with square elements except at the vicinity of the channel wall, where the mesh was refined to capture the thin film of the liquid. The computation domain was meshed with uniform square elements as shown in Fig 2. A grid independence test was carried out with four different mesh sizes, viz. 300000, 480000, 720000, and 920000 elements and the comparison of film thickness with grid size is listed in Table 2. From the table, it is observed that $\%$ of film thickness deviation was low between 720000 and 920000 elements grid. Figure 3 shows the droplet shape with four different mesh sizes at a capillary number of 0.005346 . It is evident that droplet shapes are almost similar for the mesh with 720000 and 920000 elements. Therefore, a mesh having 720000 elements was chosen in the present study to save computational time.

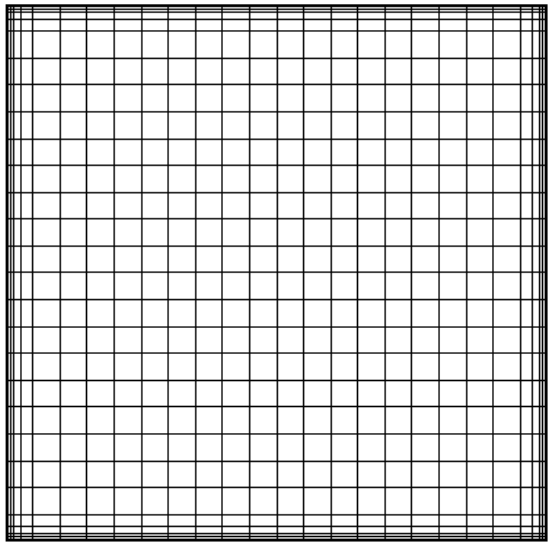

Fig. 2. Computational mesh of square microchannel.

The present work was validated with experimental correlations provided in the literature. The film thickness obtained from the present numerical model was compared with empirical correlations of Bretherton (1961), Aussillous and Quere (2000), and Han and Shikazono (2009). The film thickness values attained from the present model and empirical correlations are listed in Table 3. The film thickness attained from this study and the film thicknesses calculated from the correlations were found to be in good agreement while the highest deviation in the film thickness of the present numerical model with experimental correlations was only $7 \%$.

Table 2 Comparison of liquid film thickness with grid size.

\begin{tabular}{|l|c|c|c|c|}
\hline & 300000 elements & 480000 elements & 720000 elements & 920000 elements \\
\hline Film thickness $(\mu \mathrm{m})$ & 2.055 & 1.981 & 1.902 & 1.878 \\
\hline
\end{tabular}

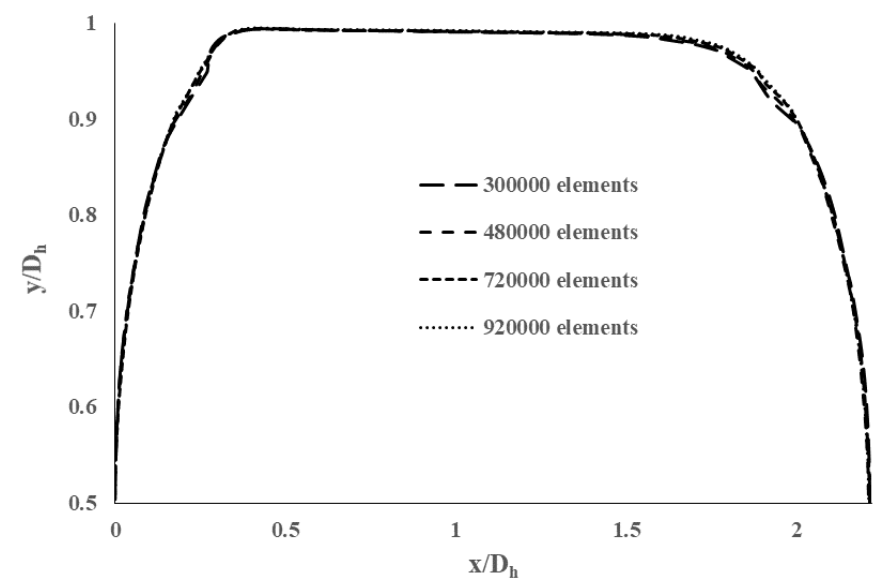

Fig. 3. Grid independence study.

Table 3 Comparison of film thickness calculated from the correlations and present study.

\begin{tabular}{|c|c|c|c|c|}
\hline Present study & Bretherton & Aussillous \& Quere & Han\& Shikazono & Deviation \% \\
\hline 0.019019 & 0.02048 & 0.01858 & 0.018658 & $2.3-7.6$ \\
\hline
\end{tabular}




\subsection{Axial Pressure Distribution}

Figure 4 shows the axial pressure variation in a square microchannel $(\mathrm{AR}=1)$ for different thermal boundary configurations. For all the thermal boundary cases, the pressure linearly varies in the slug region while at the rear interface of the droplet, the pressure jumps because of the Laplace pressure difference between the droplet and liquid slug. The pressure varies linearly in the droplet region while at the front interface of the droplet, the pressure falls and follows the liquid-only flow path. From the figure, it was evident that thermal boundary condition exercised no influence on the pressure drop

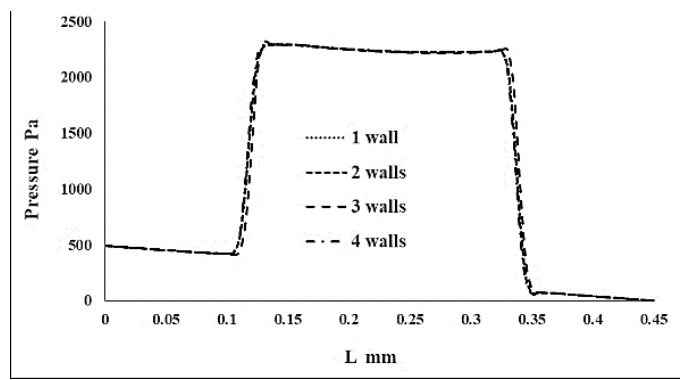

Fig. 4. Pressure distribution for different wall thermal conditions in a unit cell.

\subsection{Heat Transfer}

In this work, rate of heat transfer is represented by the Nusselt number $(\mathrm{Nu})$, and under uniform heat flux, $N u$ is given by Eq. 7 (Walsh et al. 2010).

$$
N u=\frac{q_{w} d}{k\left(T_{w}-T_{b}\right)}=\frac{h_{c} D_{h}}{k}
$$

In two-phase flows, dispersed phase fluid is separated by continuous phase liquid slugs. The fluid movement in the droplet is restricted by the front interface of the droplet, because of which the fluid changes its direction towards the channel wall. The fluid takes the heat from the channel wall and moves towards the rear interface of the droplet. At the rear interface, the fluid flow direction changes again to the center of the droplet. Hence, recirculation is produced by the droplet rear interfaces. The recirculation within fluids improves the thermal performance of the two-phase flows over singlephase flow. The recirculation zone in a unit cell has been shown in Fig. 6. Internal recirculation can be seen in the continuous phase, while the dispersed phase has three types of recirculation zones. The large one at the middle of the droplet and the other two are at the interfaces of the liquid droplet. These recirculations improve the thermal performance of liquid-liquid flows whereas, in gas-liquid flows, these recirculations make a negligible contribution as the gas has low thermophysical properties. Similar recirculation zones were also observed by Gupta et al. (2013), and Lac and Sherwood (2009). The Nusselt number of Taylor flow through the square microchannel with all four walls subjected to in Taylor flows. The unit cell consists of one droplet fenced by two halves of the liquid slug.

The axial pressure variation in a unit cell with varying aspect ratios $(\mathrm{AR}=1,2 \& 5)$ has been shown in Fig. 5. For a fixed hydraulic diameter, an increase/decrease in the AR decreases the crosssectional area of the channel. In this study, the volume fraction of fluid was constant, so that with an increase in AR, the length of droplet increased, and the slug length decreased. The axial pressure drop of Taylor flow increased with an increase in droplet length and the same observation was reported by Jovanović et al. (2011).

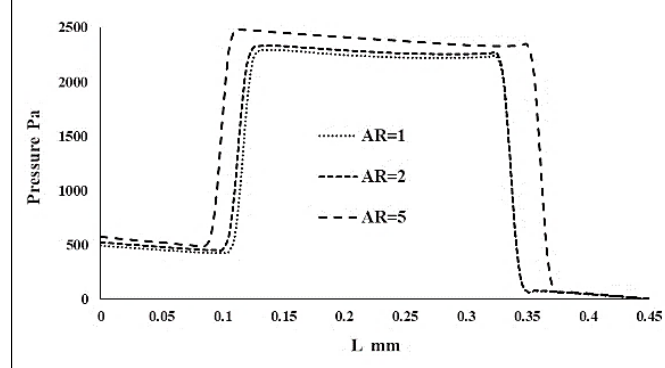

Fig. 5. Pressure distribution for varying aspect ratios in a unit cell.

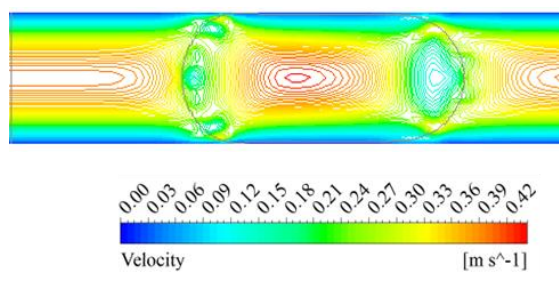

Fig. 6. Recirculation in the unit cell.

constant heat flux was found to be 9.84 and the result was compared with Leung et al. (2010) correlation shown in Eq. 8.

$N u=4.364+\frac{0.13}{\left(L_{S}^{*}\right)^{0.46} C a^{0.3}}$

Where

$L_{s}^{*}=\frac{L_{\text {slug }}}{\operatorname{Re} \cdot \operatorname{Pr} \cdot D_{h}}$

From the correlation, $N u$ was found to be 9.86, which implies that the present numerical model matches well with the existing correlation.

Thermal performance of Taylor flow was examined in a rectangular microchannel by varying $\mathrm{AR}$ of the channel in the range of 0.2 to 5 for five different thermal boundary cases. The width and height of the microchannel were taken in such a way that the hydraulic diameter of the microchannel did not vary. Figure 7 shows the rectangular channel cross-section with uniform heat flux on four walls. 


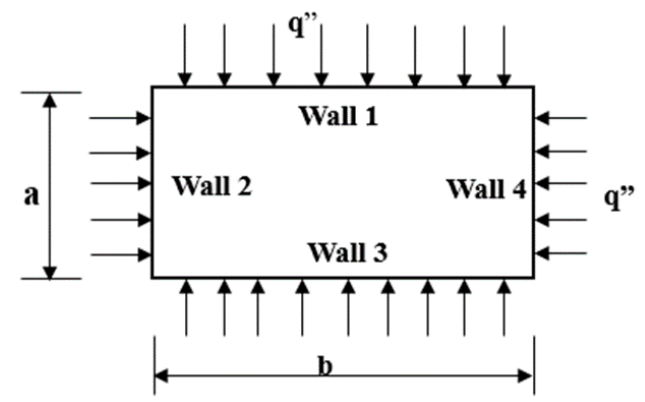

Fig. 7. Rectangular microchannel cross-section with constant heat flux on four walls.

The hydraulic diameter of the rectangular microchannel is defined as

$D_{h}=\frac{4 \cdot a \cdot b}{2(a+b)}$, and aspect ratio, $\alpha=\frac{a}{b}$

Different wall heat flux thermal boundary cases (BC) are shown in Table 4. In the first thermal BC, uniform heat flux was applied to all 4 walls of the microchannel. In the second thermal BC, wall 1 in Fig. 7 has been considered as an adiabatic wall and the remaining three walls are considered as heating walls. In opposite wall $\mathrm{BC}$, walls 1 and 3 are considered as heating walls while the other two walls were assumed to be adiabatic walls. For a single wall $\mathrm{BC}$, heat flux was applied to only wall 1 and the remaining 3 walls were considered as adiabatic walls. And for adjacent wall BC, constant heat flux was applied to walls 1 and 2 and the remaining walls were considered to be adiabatic. The wall temperature was not the same for all the thermal boundary cases for varying aspect ratios. The wall temperature for different boundary thermal boundary cases are given as (Dharaiya and Kandlikar 2012):

1. Constant heat flux on four walls

$T_{w}=\frac{T_{a}+T_{b} \cdot \alpha}{1+\alpha}$

where $T_{a}=\frac{T_{1}+T_{3}}{2}$ and $T_{b}=\frac{T_{2}+T_{4}}{2}$

2. Constant heat flux on three walls

$T_{w}=\frac{T_{a} \cdot 2 \alpha+T_{b}}{1+2 \alpha}$

where $T_{a}=\frac{T_{2}+T_{4}}{2}$ and $T_{b}=T_{3}$

3. Constant heat flux on opposite walls

$T_{w}=\frac{T_{1}+T_{3}}{2}$

4. Constant heat flux on a single wall

$T_{w}=T_{1}$

5. Constant heat flux on adjacent walls

$T_{w}=\frac{T_{1}+T_{2} \cdot \alpha}{1+\alpha}$

where $T_{1}, T_{2}, T_{3}$, and $T_{4}$ are average temperatures on walls $1,2,3$, and 4 respectively.

Table 4 Different thermal boundary cases.

\begin{tabular}{|c|c|c|c|c|c|}
\hline & $\begin{array}{c}\text { Four wall } \\
\text { BC }\end{array}$ & $\begin{array}{c}\text { Three wall } \\
\text { BC }\end{array}$ & $\begin{array}{c}\text { Opposite wall } \\
\text { BC }\end{array}$ & $\begin{array}{c}\text { Single wall } \\
\text { BC }\end{array}$ & $\begin{array}{c}\text { Adjacent wall } \\
\text { BC }\end{array}$ \\
\hline Heating walls & Walls $1,2,3, \& 4$ & Walls 2,3, \&4 & Walls $1 \& 3$ & Wall 1 & Walls $1 \& 2$ \\
\hline Adiabatic walls & -- & Wall 1 & Walls 2\&4 & Walls 2,3, \&4 & Walls 3\&4 \\
\hline
\end{tabular}

\subsubsection{Effect of Thermal Boundary Condition}

In the first thermal boundary case, the fluid takes heat equally from all 4 directions i.e., the temperature gradient is the same in all four directions. This can be seen in the temperature contour plot taken at the droplet center shown in Fig. 8(a). From the temperature contour, it has been observed that the temperature distribution is uniform except at the corners, while high temperatures regions can be seen at the corners of the microchannel, where the fluid flow is steady and film thickness is large. Under the second thermal boundary condition, the fluid absorbs heat from 3 walls but the recirculation phenomenon does not change with changes in thermal boundary conditions. From temperature contour Fig. 8(b), it can be observed that there is a high-temperature region near-wall 3 and the low-temperature region has been observed near-wall 1 as it is considered adiabatic wall, on account of which there was no heat interaction between wall 1 and the fluid. The fluid bulk temperature was lower compared to the fourwall heating case. Therefore the difference between wall and bulk temperatures decreases, which results in higher heat transfer. Under the opposite wall boundary case, it is also observed that the fluid bulk temperature is lower and the wall temperature is also reduced compared to the four-wall heating case. The fluid takes the heat from both opposite walls uniformly, so that more uniform temperature is obtained in the temperature contour shown in Fig. $8(\mathrm{c})$. Hence, the rate of heat transfer increases in the opposite heating wall cases compared to case 1 and 2 thermal boundary conditions.

Under the single wall thermal boundary condition, only one wall transfers heat to the fluid. From the temperature contour shown in Fig. 8(d), it can be seen that the high-temperature region near-wall 1 and the low-temperature (inlet temperature) region were 

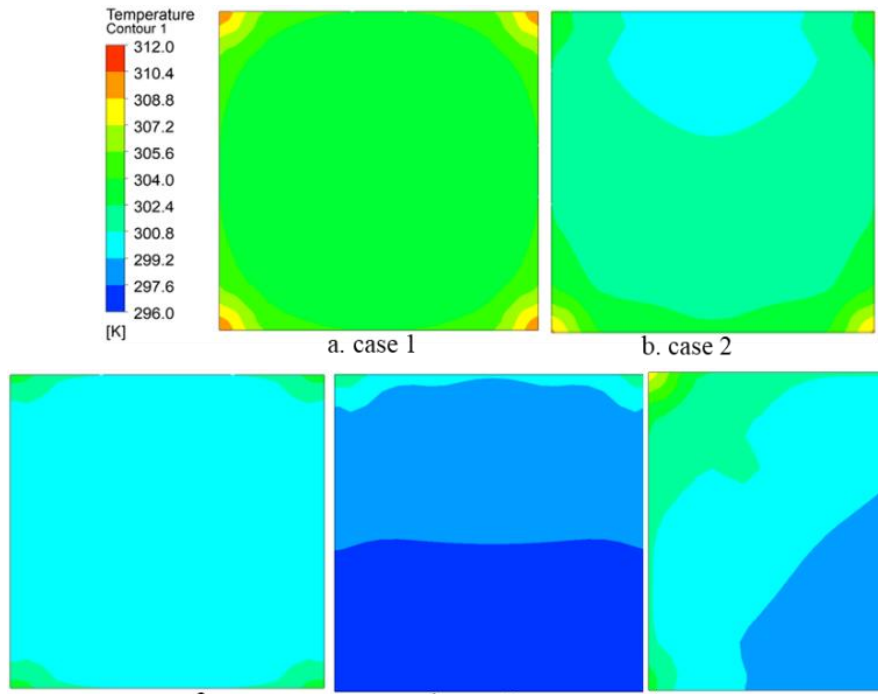

c. case 3

d. case 4

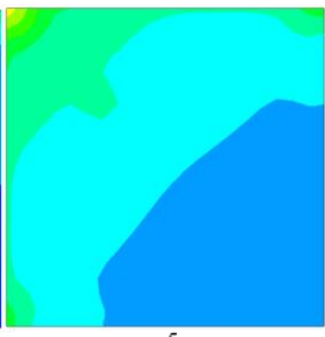

Fig. 8. Temperature contours at the middle of droplet for different thermal boundary cases.

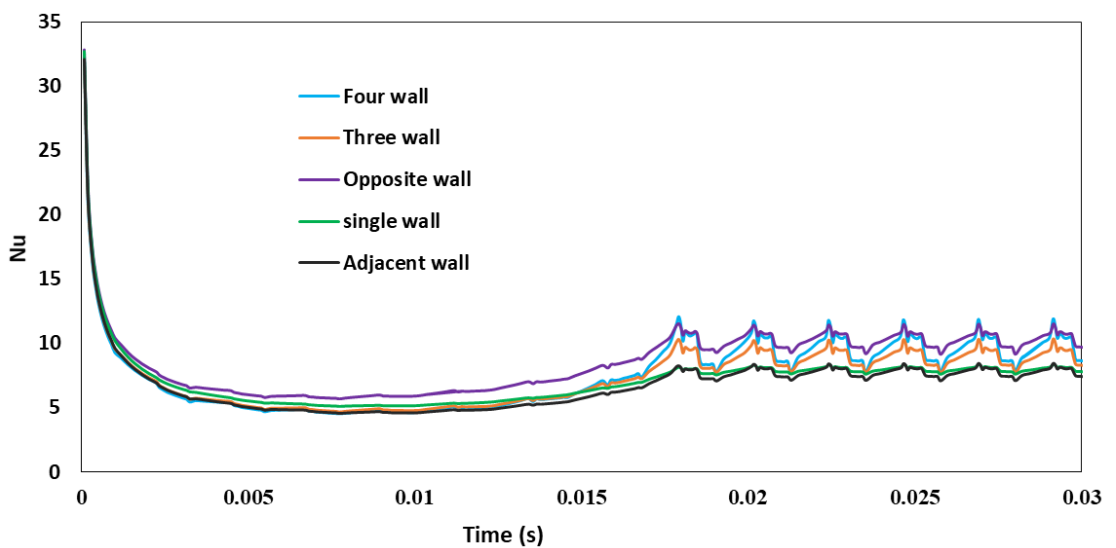

Fig. 9. Time-averaged $\mathrm{Nu}$ variation.

observed in almost half of the domain. Thus the bulk temperature is lower compared to other thermal boundary conditions, and also the difference in wall and bulk temperatures is more, which indicates lower heat transfer for single-wall thermal boundary case. In adjacent wall boundary condition, uniform heat flux is applied to adjacent walls, so that heat is transferred to the fluid from walls $1 \& 2$. From the temperature contour shown in Fig. 8(e), it can be observed that the fluid near the heating walls has more temperature with regard to the fluid near the adiabatic walls. The rate of heat transfer was found to be lower compared to other four thermal boundary cases, even though the bulk temperature of the fluid was higher than in a single wall boundary case. This is because of the higher wall temperature at the channel walls.

The time-averaged $N u$ variation in a square microchannel for all the boundary cases is shown in Fig. 9. As discussed above, $N u$ is maximum in the case of the opposite wall boundary case and it is least for the adjacent wall boundary case. The peak in time-averaged $N u$ appears when the droplet was about to leave the domain and it started to decrease while the droplet leaving the domain. Again $\mathrm{Nu}$ rises as the next droplet progresses towards the outlet of the domain. When all four walls are subjected to uniform heat flux, the heat transfer through the square microchannel $(\alpha=1)$ was validated by heat transfer correlation of Leung et al. (2010). For the same geometrical configuration, heat transfer rates for the remaining thermal boundary cases with a percentage of improvement have been shown in Table 5. These $\mathrm{Nu}$ values follow the same pattern of single-phase flow heat transfer results (Dharaiya and Kandlikar 2012). Though $N u$ is higher for the opposite wall case, more heat transfer improvement was observed in the four-wall boundary case.

\subsubsection{Effect of Aspect Ratio}

Figure 10 shows the droplet shapes and the radial velocity contours for different aspect ratios $(A R=0.2-5) .3 D$ view of droplet shapes are shown in the first column; second and third columns show the cross-sectional view of the droplet shapes and radial velocity in the $y$ and $\mathrm{z}$-directions respectively for different ARs of the microchannel. The droplet is not confined in z-direction for microchannel with $\mathrm{AR}=0.2$ and it acquires a drum shape (Fig. 10(a)). 
Table 5 Comparison of $\mathrm{Nu}$ for two-phase \& single-phase flows .

\begin{tabular}{|c|c|c|c|c|c|}
\hline $\mathrm{Nu}$ & $\begin{array}{c}\text { Four wall } \\
\text { BC }\end{array}$ & $\begin{array}{c}\text { Three wall } \\
\text { BC }\end{array}$ & $\begin{array}{c}\text { Opposite wall } \\
\text { BC }\end{array}$ & $\begin{array}{c}\text { Single wall } \\
\text { BC }\end{array}$ & $\begin{array}{c}\text { Adjacent wall } \\
\text { BC }\end{array}$ \\
\hline Two-phase flow & 9.85 & 9.08 & 10.41 & 7.99 & 7.81 \\
\hline Single phase flow & 3.47 & 3.38 & 4.69 & 3.23 & 2.88 \\
\hline \% of improvement & 283 & 268 & 222 & 247 & 271 \\
\hline
\end{tabular}

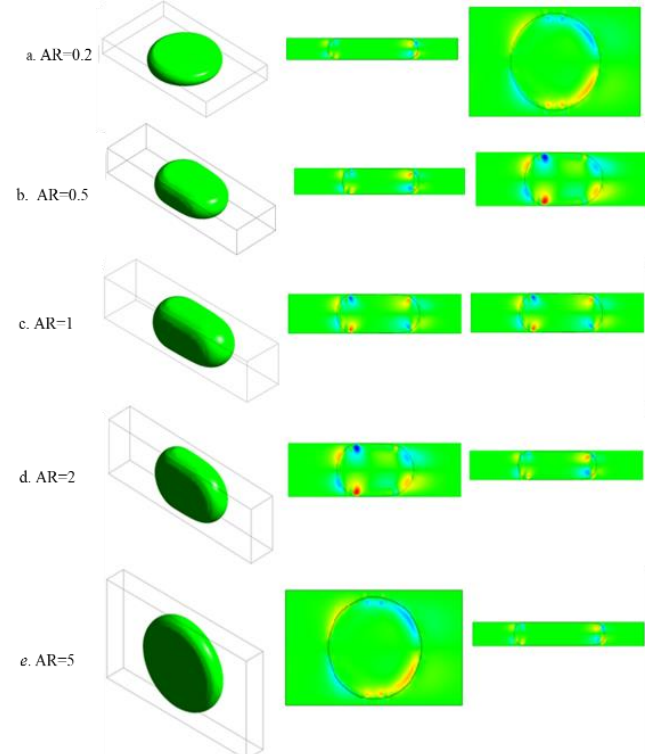

Fig. 10. Droplet shapes and the radial velocity contours in a microchannel with different aspect ratios.

The interfaces close to the top/bottom walls are flat, while the interfaces close to the side walls attain a curved shape because of the strong surface tension effect and the frail wall confinement effect. With an increase in the $\mathrm{AR}$, droplets are squeezed by the sidewalls and progressively change into bullet shape $(\mathrm{AR}=1)$, and as shown in Fig. 10(c) the interfaces near the sidewalls become flat. Due to the strong surface tension effect at the micro-levels, the droplet interfaces acquire a spherical-cap shape. The flow within the droplet forces the interface at the front cap so that the front cap has more curvature than the rear cap. With further increase in the AR, the droplet interfaces near the top/bottom walls attain a curved shape.

The recirculation inside the dispersed and continuous phase leads to considerable improvement in the heat transfer rate of Taylor flows. Radial velocity components $\left(\mathrm{u}_{\mathrm{y}}\right.$ and $\mathrm{u}_{\mathrm{z}}$ ) are the main reason for recirculation within the $3 \mathrm{D}$ microchannels. These radial velocity components are shown in Fig. 10, which reveal the presence of higher velocities at the rear and front end of the droplet, both in the dispersed and continuous phases. This occurs because of the droplet interfaces, which restricts the flow in the axial direction and induces radial velocity components. The flows on the center of the $\mathrm{Y}$ and $\mathrm{Z}$ planes are identical in square microchannels, due to the symmetry of the microchannel. With increase in AR, the confining effect of top/bottom walls decreases, and therefore these walls diminish the shear effect on the droplet. The magnitude of $u_{y}$ decreases, as a low quantity of fluid arrives at the rear interface and then flows in the radial direction. Besides, a larger channel depth needs more time for both diffusion and advection in the y-direction. Thus, the heat transfer in y-direction is repressed by the large AR. Hence, the heat transfer is lower for larger ARs than for square $(A R=1)$ microchannels. The velocity component in the $\mathrm{z}$-direction $\left(\mathrm{u}_{\mathrm{z}}\right)$ is not significantly influenced by the microchannel depth with an increase in AR, therefore heat transfer is not influenced by $\mathrm{u}_{\mathrm{z}}$. Similarly, in the case of low ARs (0.2\&0.5), the channel depth increases in z-direction and it takes more time for both diffusion and advection. Hence, the heat transfer rate is also lower for smaller aspect ratios. The same was reported by Che et al. (2015) and a similar trend was also reported by Dharaiya and Kandlikar (2012), in their study of single-phase flow heat transfer. The comparison of heat transfer rates for different thermal conditions with varying aspect ratios has been shown in Table 6 .

Figure 11 shows the time-averaged $\mathrm{Nu}$ variation for different aspect ratios of the microchannel under four-wall heating boundary conditions. As discussed, $N u$ is maximum for square microchannel, and with an increase and decrease in AR, a decrease in $N u$ can be observed. For the four-wall thermal boundary condition, the $N u$ variation is the similar for the ARs of $0.2 \& 5$ and ARs of $0.5 \& 2$.

Table 6 Comparison of $\mathrm{Nu}$ for different thermal conditions with varying aspect ratios.

\begin{tabular}{|c|c|c|c|c|c|}
\hline AR & Four wall BC & Three wall BC & Opposite wall BC & Single wall BC & Adjacent wall BC \\
\hline 0.20 & 6.548 & 5.371 & 22.819 & 10.574 & 7.297 \\
\hline 0.50 & 8.996 & 8.268 & 12.005 & 9.108 & 7.924 \\
\hline 1.00 & 9.847 & 9.081 & 10.410 & 7.995 & 7.812 \\
\hline 2.00 & 9.006 & 10.095 & 8.604 & 6.996 & 8.074 \\
\hline 5.00 & 6.548 & 12.174 & 3.796 & 3.590 & 7.313 \\
\hline
\end{tabular}




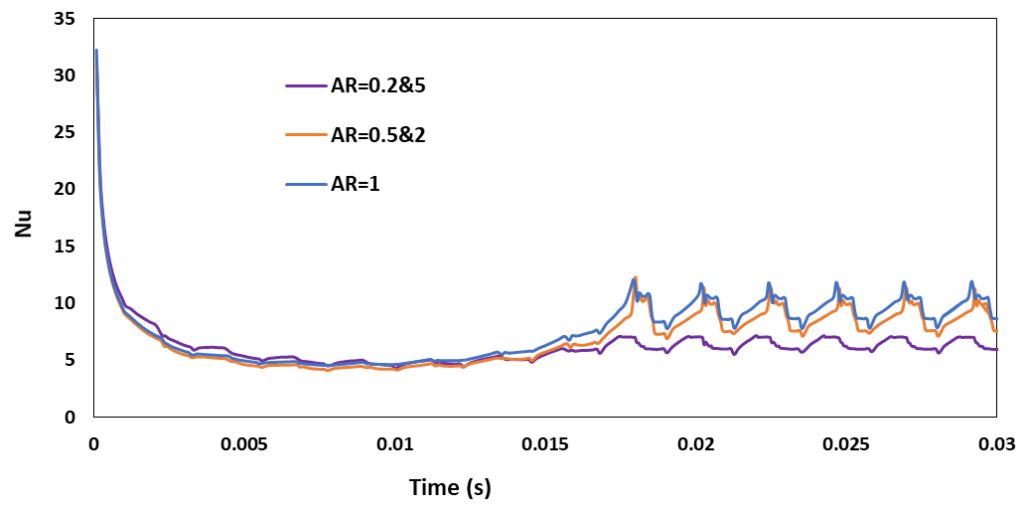

Fig. 11. Time-averaged $N u$ variation for different aspect ratios of the microchannel under four-wall boundary case.
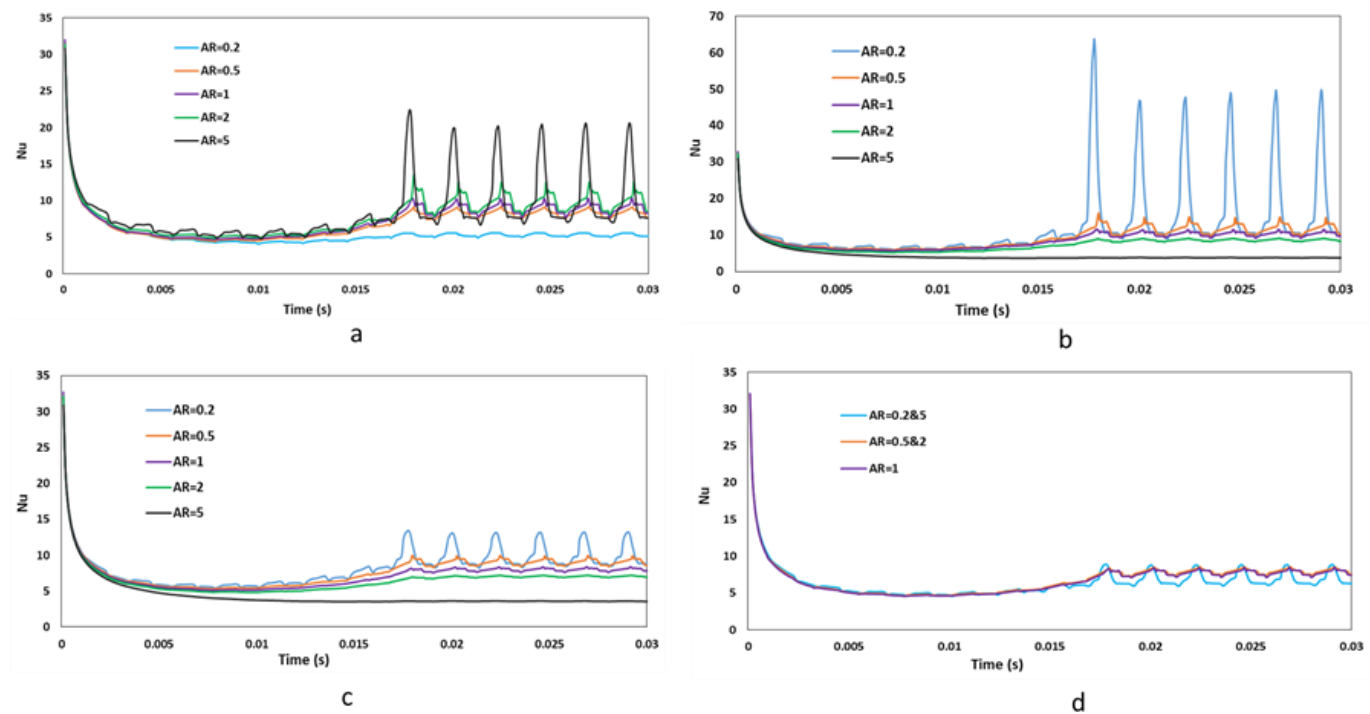

Fig. 12. Time-averaged $\mathrm{Nu}$ variation for different aspect ratios of the microchannel a) three walls, b) opposite walls, c) single wall, and d) adjacent walls boundary cases.

As discussed above, microchannels with $\mathrm{AR}=0.2$ \& 0.5 under the second thermal boundary case also microchannel has more channel depth in the zdirection and require more time for both advection and diffusion. And, the longer wall (i.e., wall 1) has been considered as adiabatic in y-direction, and thus the rate of heat transfer is smaller compared to the four-wall thermal boundary condition.

For microchannels of $\mathrm{AR}=2 \& 5$, the adiabatic wall (wall 1) is smaller compared to low AR microchannel walls, and hence the heating area is increasing with an increase in the AR. An increase in the heating area leads to an increase in the bulk temperature, which results in heat transfer augmentation. Therefore the heat transfer rate increases with an increase in AR under case 2 thermal boundary conditions, the same has been reported by Dharaiya and Kandlikar (2012). The same can be observed from the time-averaged $\mathrm{Nu}$ variation graph shown in Fig. 12(a). The spike can be seen for $\mathrm{Nu}$ variation of $\mathrm{AR}=5$ as it has higher heating area.
In the case of opposite wall thermal boundary condition, walls $2 \& 4$ are considered as adiabatic walls, so that there is no heat transfer in z-direction. As AR increases, the channel depth increases in ydirection, which leads to diminution in heat transfer rate. Therefore, the overall heat transfer decreases with an increase in AR of the microchannel. The same can be seen from the time-averaged $N u$ variation graph shown in Fig. 12(b). Similarly, for single-wall boundary conditions also, heat transfer decreases with an increase in AR of microchannel as only wall 1 is considered as the heating wall. The Time-Averaged $\mathrm{Nu}$ variation graph under a single wall thermal boundary case has been shown in Fig. 12(c). For adjacent wall thermal boundary condition, walls $1 \& 2$ are assumed to be heating walls. As discussed in four-wall boundary condition, more time is required for diffusion and advection in $\mathrm{z}-$ direction when the AR of the microchannel is 0.2 . With an increase in AR, the channel depth decreases in $\mathrm{z}$-direction, so that heat transfer rate increases for AR of 0.5. For square microchannel, the depth of the channel is equal in both directions but the heating 
area was small compared to AR of 0.5 so that $\mathrm{Nu}$ decrease a little. For a channel with an AR of 2, the $N u$ increases a little and with further increase in AR, the channel depth increases in y-direction, which leads to decrease in heat transfer rate. Time-averaged $\mathrm{Nu}$ variation graph under adjacent wall boundary case for all ARs has been shown in Fig. 12(d). Nu was observed to be higher for $0.5 \& 2$ ARs and was least for $0.2 \& 5$ ARs of the microchannel.

\section{CONCLUSIONS}

Numerical simulations were performed on liquidliquid Taylor flow and heat transfer in a 3D microchannel with uniform wall heat flux boundary conditions. Dodecane and water were considered as primary and secondary phases, and the two-phase velocity was taken as $0.2 \mathrm{~m} / \mathrm{s}$. The thin film between the wall and droplet interface was compared with empirical correlations and it matches well with the correlations. Taylor flow heat transfer was studied with varying aspect ratios of the microchannel in the range of 0.2 to 5 under five different thermal boundary cases. Pressure drop in the Taylor flow does not change with thermal boundary condition, but with an increase in AR, the cross-sectional area of the microchannel reduces, because which the axial pressure drop increases. Taylor flow heat transfer through the square $(\mathrm{AR}=1)$ microchannel with fourwall boundary case has been compared with the experimental correlation and found to match precisely. A higher $N u$ (10.41) was found when the opposite walls were conducting heat and $N u(7.81)$ was minimum when the adjacent walls were subjected to uniform heat flux. Under four wall and adjacent wall boundary conditions, the maximum heat transfer rate was obtained for square microchannel as the channel depth was equal in both y \& z-directions. An increase in AR (2\&5) channel depth led to an increase in y-direction, requiring more time for advection and diffusion; hence the heat transfer rate decreased, and with decreasing AR $(0.5 \& 0.2)$, the depth of channel increased in $\mathrm{z}$ direction. For case 2 thermal boundary conditions, the heat transfer rate augmented with an increase in $\mathrm{AR}$, and for case 3 and 4 boundary conditions, the heat transfer diminished with an increase in AR.

\section{REFERENCES}

Abdollahi, A., S. E. Norris and R. N. Sharma. (2020). Fluid Flow and Heat Transfer of Liquid-Liquid Taylor Flow in Square Microchannels. Applied Thermal Engineering 172 (115123), 1-13.

Asadolahi, A. N., R. Gupta, D. F. Fletcher and B. S. Haynes (2011). CFD Approaches for the Simulation of Hydrodynamics and Heat Transfer in Taylor Flow. Chemical Engineering Science 66(22), 5575-84.

Asthana, A., I. Zinovik, C. Weinmueller and D. Poulikakos. (2011). Significant Nusselt Number Increase in Microchannels with a Segmented Flow of Two Immiscible Liquids: An Experimental Study. International Journal of Heat and Mass Transfer 54(7-8), 1456-64.

Aussillous, P. and D. Quere. (2000). Quick Deposition of a Fluid on the Wall of a Tube. Physics of Fluids 12(10), 2367-71.

Bandara, T., S. C. Cheung and G. Rosengarten (2015). Slug Flow Heat Transfer in Microchannels: A Numerical Study. Computational Thermal Sciences 7(1), 81-92.

Brackbill, J. U., D. B. Kothe and C. Zemach (1992). A Continuum Method for Modeling Surface Tension. Journal of Computational Physics 335-354.

Bretherton, F. P. (1961). The Motion of Long Bubbles in Tubes. Journal of Fluid Mechanics 10(2), 166-88.

Che, Z., T. N. Wong and N. T. Nguyen (2012). Heat Transfer Enhancement by Recirculating Flow within Liquid Plugs in Microchannels. International Journal of Heat and Mass Transfer 55(7-8), 1947-56.

Che, Z., T. N. Wong, N. T. Nguyen and C. Yang (2015). Three Dimensional Features of Convective Heat Transfer in Droplet-Based Microchannel Heat Sinks. International Journal of Heat and Mass Transfer 86, 455-64.

Dai, Z., Z. Guo, D. F. Fletcher and B. S. Haynes (2015). Taylor Flow Heat Transfer in Microchannels - Unification of Liquid Liquid and Gas - Liquid Results 138, 140-52.

Dharaiya, V. V. and S. G. Kandlikar (2012). Numerical Investigation of Heat Transfer in Rectangular Microchannels under H2 Boundary Condition during Developing and Fully Developed Laminar Flow. Journal of Heat Transfer 134(2), 1-10.

Eain, M. M. G., V. Egan and J. Punch (2013). Film Thickness Measurements in Liquid-Liquid Slug Flow Regimes. International Journal of Heat and Fluid Flow 44, 515-23.

Eain, M. M. G., V. Egan and J. Punch (2015). Local Nusselt Number Enhancements in LiquidLiquid Taylor Flows. International Journal of Heat and Mass Transfer 80, 85-97.

Gupta, R. S. S. Leung, R. Manica, D. F. Fletcher and B. S. Haynes (2013). Hydrodynamics of Liquid-Liquid Taylor Flow in Microchannels. Chemical Engineering Science 92, 180-89.

Gupta, R., D. F. Fletcher and B. S. Haynes (2009). On the CFD Modelling of Taylor Flow in Microchannels. Chemical Engineering Science 64(12), 2941-50.

Gupta, R., D. F. Fletcher and B. S. Haynes (2010). CFD Modelling of Flow and Heat Transfer in the Taylor Flow Regime. Chemical Engineering Science 65(6), 2094-2107.

Han, Y. and N. Shikazono (2009). Measurement of the Liquid Film Thickness in Micro Tube Slug Flow. International Journal of Heat and Fluid 
S. Chandrasekhar and V. R. K. Raju / JAFM, Vol. 14, No. 6, pp. 1755-1765, 2021.

Flow 30(5), 842-53.

Irandoust, S. and B. Andersson. (1989). Liquid Film in Taylor Flow through a Capillary. Industrial and Engineering Chemistry Research 28(11), 1684-88.

Jovanović, J. W. Zhou, E. V. Rebrov, T. A. Nijhuis, V. Hessel and J. C. Schouten (2011). LiquidLiquid Slug Flow: Hydrodynamics and Pressure Drop. Chemical Engineering Science 66(1), 42-54.

Kreutzer, M. T. F. Kapteijn, J. A. Moulijn, C. R. Kleijn and J. J. Heiszwolf (2005). Inertial and Interfacial Effects on Pressure Drop of Taylor Flow in Capillaries. AIChE Journal 51(9), 2428-40.

Lac, E. and J. D. Sherwood (2009). Motion of a Drop along the Centreline of a Capillary in a Pressure-Driven Flow. Journal of Fluid Mechanics 640, 27-54.

Leung, S. S. Y., R. Gupta, D. F. Fletcher and B. S. Haynes (2012). Effect of Flow Characteristics on Taylor Flow Heat Transfer. Industrial and Engineering Chemistry Research 51(4), 201020.

Leung, S. S. Y., Y. Liu, D. F. Fletcher and B. S. Haynes (2010). Heat Transfer in WellCharacterised Taylor Flow. Chemical Engineering Science 65(24), 6379-88.

Liu, H., C. O. Vandu and R. Krishna (2005). Hydrodynamics of Taylor Flow in Vertical Capillaries: Flow Regimes, Bubble Rise Velocity, Liquid Slug Length, and Pressure Drop. Industrial and Engineering Chemistry Research 44(14), 4884-97.

Muzychka, Y. S., E. J. Walsh and P. Walsh (2010).
Heat Transfer Enhancement Using Laminar Gas-Liquid Segmented Fluid Streams. Proceedings of the ASME InterPack Conference 2009, IPACK2009 2(April), 26172.

Triplett, K. A., S. M. Ghiaasiaan, S. I. Abdel-Khalik and D. L. Sadowski (1999). Gas-Liquid TwoPhase Flow in Microchannels Part I: Two-Phase Flow Patterns. International Journal of Multiphase Flow 25(3), 377-94.

Urbant, P., A. Leshansky and Y. Halupovich (2008). On the Forced Convective Heat Transport in a Droplet-Laden Flow in Microchannels. Microfluidics and Nanofluidics 4(6), 533-42.

Vivekanand, S. V. B. and V. R. K. Raju (2020). Modulated Wall Motion Approach for Augmenting Slug Flow Heat Transfer between Two Micro-Parallel Plates. Physics of Fluids 32(3), 032001-22.

Vivekanand, S. V. B. and V. R. K. Raju (2019). Effect of Wall Temperature Modulation on the Heat Transfer Characteristics of Droplet-Train Flow inside a Rectangular Microchannel. Chinese Journal of Chemical Engineering.

Walsh, P. A., E. J. Walsh and Y. S. Muzychka (2010). Heat Transfer Model for Gas-Liquid Slug Flows under Constant Flux. International Journal of Heat and Mass Transfer 53(15-16), 3193-3201.

Warnier, M. J. F., M. H. J. M. De Croon, E. V. Rebrov and J. C. Schouten (2010). Pressure Drop of Gas-Liquid Taylor Flow in Round Micro-Capillaries for Low to Intermediate Reynolds Numbers. Microfluidics and Nanofluidics 8(1), 33-45. 